\title{
Stimulation cérébrale profonde et déplacement d'électrode: évaluation à moyen terme
}

\author{
Caire F., Coste J., Gabrillargues J., Chabert E., \\ Claise B., Derost P., Durif F., Lemaire J.-J.
}

Adresse : Hôpital Gabriel-Montpied, Clermont-Ferrand, France

Introduction. Un éventuel déplacement des électrodes de stimulation cérébrale profonde (DBS), à distance de l'implantation, est évoqué pour expliquer certaines discordances entre effets bénéfiques per-opératoires et en stimulation chronique. Nous avons évalué ce déplacement en nous appuyant sur des données d'IRM postopératoires tardives.

Matériel-Méthode. Neuf patients ayant bénéficié de la mise en place bilatérale d'électrodes DBS pour maladie de Parkinson idiopathique sévère avec de bons résultats cliniques (amélioration motrice $>60 \%$ ) ont été inclus. Nous nous sommes intéressés aux contacts utilisés en stimulation chronique (contacts effectifs, stimulation monopolaire). Les positions initiales de ces contacts $(n=18)$ ainsi que des électrodes $(n=18)$ ont été déterminées à partir de clichés téléradiographiques de fin de procédure (électrodes fixées, cadre en place) et projetées (correspondance stéréotaxique) sur I'IRM pré-opératoire stéréotaxique (pondération T2, cadre en place). Une IRM réalisée à distance (pondération T1, délai de 6 à 12 mois) a été mise en correspondance avec l'IRM pré-opératoire (algorithme d'optimisation de voxel à voxel). Pour chacun des contacts effectifs, nous avons mesuré la distance entre le centre du contact identifié en radiographie et projeté sur l'IRM pré-opératoire et celui déterminé à partir de l'artefact sur l'IRM à distance $(\mathrm{dc})$.

Résultats ou Cas rapporté. Dans tous les cas, la position de l'électrode, relevée sur les clichés téléradiographiques, était située dans les limites de l'artéfact de l'électrode sur l'IRM tardive. La distance moyenne entre les positions initiales et tardives des centres des contacts (dc) était de 1,3 $\mathrm{mm}( \pm 0,5 \mathrm{~mm})$.

Conclusion. En prenant en compte les différentes incertitudes liées aux modes de recueil des coordonnées (téléradiographie et IRM), nos résultats ne semblent pas montrer de différence significative entre la position initiale des contacts effectifs et celle mesurée tardivement. Ces résultats sont discutés en fonction des données de la littérature. 